\title{
Enhancement of the Thermal Performance Characteristics of an Electrical Power Transformer
}

\author{
Nawras Mohammed Azbar, Hayder Mohammad Jaffal ${ }^{*}$, Basim Freegah \\ Mechanical Engineering Department, College of Engineering, Mustansiriyah University, Baghdad, Iraq \\ E-mail: jaffal.env@uomustansiriyah.edu.iq
}

Received: 15 June 2020; Revised: 29 June 2020; Accepted: 23 July 2020

\begin{abstract}
A 3D numerical simulation was conducted to test the effects of the geometrical and operational parameters on the cooling performance of a three-phase electrical distribution transformer (250 kVA oil natural air natural (ONAN)). The geometric parameters include the shape of the transformer (rectangular, circular, and hexagonal), fins shape (rectangular, semicircular, and trapezoidal) as well it arrangement (asymmetric fin heights and perforated fins). Both of oil temperature and thermal load have been used as boundary conditions. In order to verify the reliability of the numerical model, comparison between numerical results and experimental finding has been done. The results have indicated that the circular and hexagonal shapes reduced the average oil temperature by $3.4 \%$ and $4.7 \%$, respectively, compared to the traditional transformer shape (rectangular). Furthermore, the lowest average oil temperature was observed for the trapezoidal fin, followed by the rectangular and semicircular fins. Additionally, it has been noticed that the asymmetric fin heights of the trapezoidal and perforated trapezoidal fins been contributed to the improvement of the cooling performance of the transformer. Furthermore, the best thermal performance was obtained with the trapezoidal perforated fin to compared other arrangement of fins. Finally, the highest reduction in oil has been obtained by the use of hexagonal transformer with a perforated trapezoidal fin approximately by $12 \%$ compared to traditional rectangular transformer. Hence, it can be concluded that the shape of the transformer and fins play an important role in thermal performance of such systems.
\end{abstract}

Keywords: power transformer, numerical simulation, natural convection, thermal optimization, fin shape, transformer geometry

\section{Introduction}

Power transformers are one of the important basic equipment for electrical power distribution and transmission systems. During the conversion of electricity in transformers, the temperature rises; this condition rise directly affects the performance of the electrical transformers, their average life, maintenance costs, in addition to their risks, such as combustion to which they are exposed [1]-[4]. The efficiency of the electric transformer is greatly reduced when the maximum temperature is reached. Accordingly, the transformer faces many challenges. The unwanted heat is dissipated into the atmosphere by the coolant via the fins to reduce the overheating of the transformer [5]-[8]. The cooling system used for heat dissipation during the operation directly affects the performance and life of the power transformer [9].

Copyright (C2020 Hayder Mohammad Jaffal, et al

DOI: https://doi.org/10.37256/est.212021487

This is an open-access article distributed under a CC BY license

(Creative Commons Attribution 4.0 International License)

https://creativecommons.org/licenses/by/4.0/ 
Recently, the topic of improving the cooling system of power transformers has posed a real challenge to many researchers. Tsili et al. [10] numerically modeled the hydro-thermal performance characteristics of an oil natural air natural (ONAN) transformer. The prediction of fluid flow and heat transfer characteristics of the transformer has been conducted using the finite element technique. In a specific transformer, the proposed technique provided an integrated simulation tool than can perform a detailed thermal distribution without having to know the values of the temperature gradient. Mahdi et al. [11] numerically investigated the effect of fin shape on the thermal performance of a power transformer. The temperature distribution of the power transformer for the four proposed fin shapes (rectangular shape with circular/rectangular perforator and parallelogram shape from the top/bottom sides) is compared with that of the traditional rectangular fin shape. The transformer with a parallelogram shape from the top showed the best thermal performance among the five fin shapes. Bachinger and Hamberger [12] experimentally studied the thermal characteristics of a power transformer under very low ambient temperatures $\left(-30^{\circ} \mathrm{C}\right.$ to $\left.-50^{\circ} \mathrm{C}\right)$ and different loads. An inverse relationship between the temperature of the transformer and the ambient air temperature was observed under a constant load. The use of fans to enhance the cooling resulted in higher rather than lower temperatures. Hajidavalloo and Mohamadianfard [13] experimentally conducted tests in two methods to measure the effect of solar radiation on the performance of the electrical transformer. A mathematical modeling was performed to examine the performance of the transformer under direct and indirect radiation conditions. The modeling results were compared with the experimental findings. The results indicated that the use of sun shield affects the reduction of the oil temperature. Accordingly, the lifespan of the transformer increases by more than $24 \%$. Sathyanarayana et al. [14] used an improved model to demonstrate the effect of increased ambient temperature on the lifespan of the transformer. In cases studied within 100 years, an increase in the ambient temperature by $3.5^{\circ} \mathrm{C}$ caused a reduction in the calculated age of the transformer (3-6 years). Gastelurrutia et al. [15] numerically simulated the heat transfer characteristics of ONAN power transformers. In the predicted results, the effect of turbulence modeling was assessed. This computational model can be utilized to study the natural heat transfer of oil inside the transformer and its correlation with the outdoor ambient air. Abdolzadeh et al. [16] numerically studied the heat transfer characteristics of a power transformer inside a closed space under different operating parameters. The results showed that the closed space causes a decrease in the efficiency of the transformer despite the low energy loss due to the lack of ventilation, as found in the transformers externally installed without a closed space. Farhan et al. [17] numerically investigated the effect of fin shape and nanoparticles on the thermal performance of the power transformer. The power transformer heat transfer and fluid flow of different rectangular fins with curved corners and conical fins were compared with the traditional rectangular fin. The results indicated that the curved corners can reduce the life of the transformer. Meanwhile, the conical fins demonstrated the best performance compared with the conventional fins. Arguence and Cadoux [18] analytically determined the sizes of the power transformers at the planning stage by using loads and the hot spot temperature of the distribution transformers. The practical value of this method was evaluated by estimating the increase in the host capacity of the studied transformer using a real-world data set. Wakil et al. [19] numerically studied the influence of the transformer configurations and the effect of the oil flow rate at the inlet on the thermal performance of the power transformer. Different engineering configurations and oil cooling flow rates were tested to improve the cooling of the power transformer. The results indicated that the increase in oil mixing reflected the optimal homogeneity of temperatures and the best heat transfer. Meanwhile, the increase in the flow rate of the oil in the inlet contributed to the increase in the cost without any improvement on the heat transfer due to the configuration complexity. Gong et al. [20] numerically and experimentally investigated the heat transfer and fluid flow of the $31.5 \mathrm{MVA} / 110 \mathrm{kV}$ ONAN power transformer. At different load values, the mathematical model using the finite volume technique has been applied to calculate the hottest point on the power transformer. Chereches et al. [21] numerically investigated the effect of the inlet flow rate on the thermal performance of a 40 MVA power transformer with different cooling channel designs. In this transformer, the forced convection for the air and oil was adopted, and a pump was used to circulate the oil and fan to ventilate the air in the radiators. The results showed that the use of an obstacle placed near the inlet of the transformer improved the performance of the transformer because it helped direct the oil flow and cooled the active parts. Kim et al. [22] numerically and experimentally investigated the performance characteristics of two types of power transformer: oil natural air natural (ONAN) and oil-directed forced air natural (ODAN). A comparison of the performance characteristics between the ONAN and the ODAN transformers was conducted on the basis of the cooling capacity, the temperature difference between the top and the bottom of the transformer, and the distribution of the coolant temperatures. The 
results showed a $20.1 \%$ improvement in the thermal performance when ODAN was used instead of ONAN. The performance enhancement of the power transformer has been studied numerically and experimentally by Gamil et al. [23]. Numerically, the finite element technique was used to predict the transformer characteristics in terms of heat transfer and fluid flow. They have been reported that the prediction accuracy of the transformer characteristics, such as, the hot spot temperature and transformer life are depending mainly on the accuracy of analyzing the thermal losses of transformer components. Hannun et al. [24] carried out a numerical study to the improve the power transformer performance using external air heat exchanger. The effects of several external heat exchanger parameters (pipe diameter, pipe length, and air inlet velocity) on decreasing the average oil temperature have been studied. The results have been shown that the performance of the power transformer improved effectively when using the earth air heat exchanger. It was noticed that the oil temperature decreased by $18.5^{\circ} \mathrm{C}$ under the boundary conditions of this study. The effect of oil characteristics on the enhancement of power transformer thermal performance has been investigated numerically by Hasan [25]. In this study, a new oil as coolant for the power transformers has been used and compared it to a conventional transformer oil. The suggested coolant is an oil mixed with paraffin wax as a variable phase substance with (5-25\%) volume concentrations. The obtained results showed that the addition of phase change of paraffin wax transformer to the oil contributes to improving the transformer thermal performance by decreasing the oil temperature and hence increased transformer life.

Many electrical transformers experience constant failure in the summer due to the hot climate conditions. As well as, the literature review indicated clearly that the high temperature is the main factor that have a significant effect on the efficiency of the electrical transformers. So, the main aim of the current study to improve the performance of electrical transformers in countries with hot climate conditions to prevent the transformers from collapsing, extend their lifespan, and reduce their maintenance. As this study focuses on using passive heat transfer enhancement techniques to improve the thermal performance of the electrical transformer. The effect of different shapes of the transformer along with various the shape and arrangement of the fins have been investigated on the cooling performance of the electrical transformer.

\section{Numerical methodology}

\subsection{Physical model}

Figure 1(a) shows a real picture of a three-phase power distribution transformer (250 kVA ONAN) used in the study. Figure 1(b) shows the schematic of the transformer.

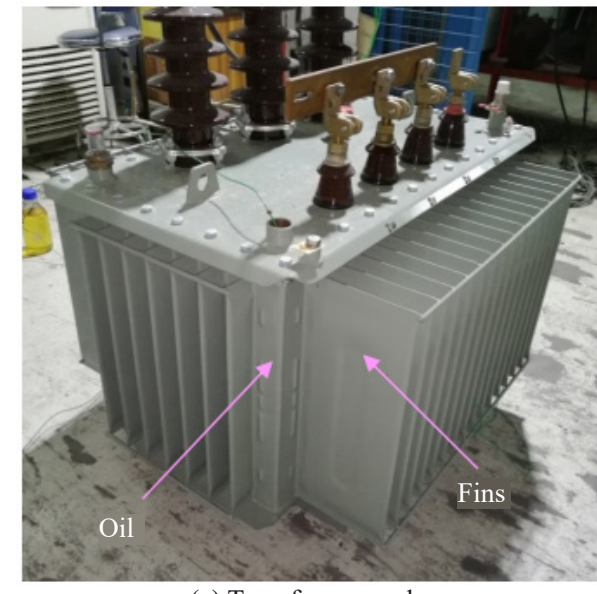

(a) Transformer real

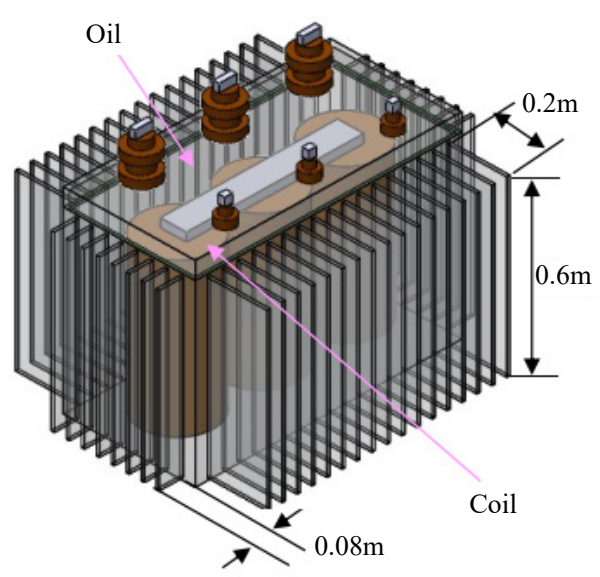

(b) Transformer computational

Figure 1. $250 \mathrm{kVA}$ ONAN traditional electrical power transformer 
The details of the dimensions of the ONAN distribution transformer used in this study are illustrated in Table 1. The electrical coils are stationed at the center of the transformer. The distance between the coils and the body of the external transformer is filled with electrical transformer oil, which is the medium that transfers the heat generated from the coils to the ambient air through the fins. The mutual induction between two circuits associated with a common magnetic flux is the material basis of the transformer. In the internal structures of the transformer, the temperature rises due to the heat generated during its operation. In general, transformers that operate at a low efficiency have high temperatures due to large losses. Meanwhile, efficient transformers have low temperatures and losses.

Table 1. The electrical coils are stationed at the center of the transformer

\begin{tabular}{cc}
\hline Descriptions & Dimension \\
\hline Casing height & $0.7 \mathrm{~m}$ \\
Casing length & $0.9 \mathrm{~m}$ \\
Casing width & $0.45 \mathrm{~m}$ \\
Fin height & $0.6 \mathrm{~m}$ \\
Fin length & 0.2 and $008 \mathrm{~m}$ \\
Total number of fins & 50 \\
Space between fins & $0.04 \mathrm{~m}$ \\
Total fins area & $9.6 \mathrm{~m}^{2}$ \\
Total transformer area & $12.3 \mathrm{~m}^{2}$ \\
\hline
\end{tabular}

\subsection{Proposed transformer geometry and fin shape}

A comparison study has been conducted on the several proposed changes in the geometry of the transformer that may affect heat transfer process to improve the performance of the conventional electrical transformers. The proposed designs may allow additional heat to spread to the ambient air while maintaining the transformer outer circumference. Two geometries of the electrical transformers (circular and hexagonal) with the same surface area as conventional rectangular transformers $\left(12.3 \mathrm{~m}^{2}\right)$ are suggested. Figure 2 shows the traditional transformer and the new proposed transformer geometries with their important dimensions. The shape of the fins and their distribution around the circumference of the transformer directly affect the performance and the heat dissipation from the oil to the outer circumference of the transformer. Accordingly, the performance of the transformer with semicircular trapezoidal fins is compared with that of the transformer with a traditional rectangular fin shape. The effect of the arrangement of the trapezoidal fins (asymmetric fin heights and perforated fins) on the hexagonal transformer is examined. The total fins area with the same surface is $9.6 \mathrm{~m}^{2}$. Figure 3 shows the proposed fin shapes.

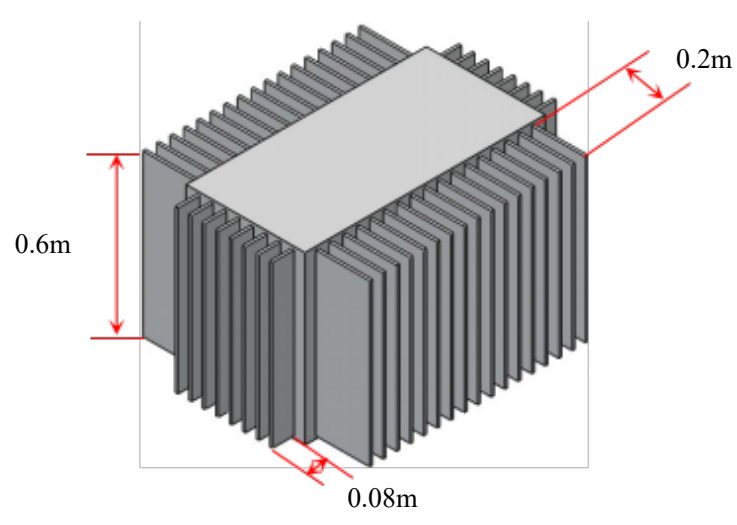

(a) Rectangular transformer geometry 


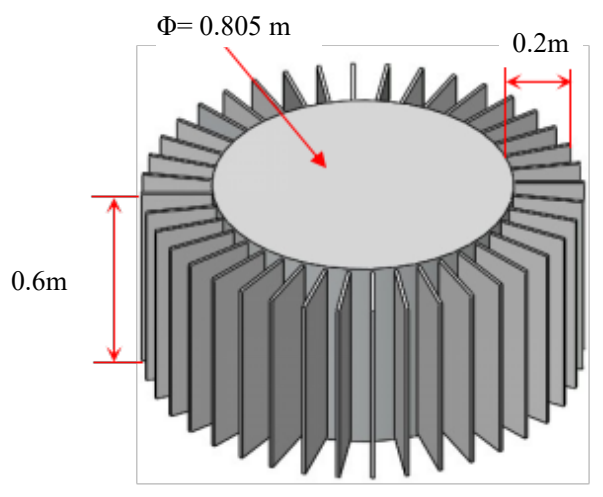

(b) Circular transformer geometry

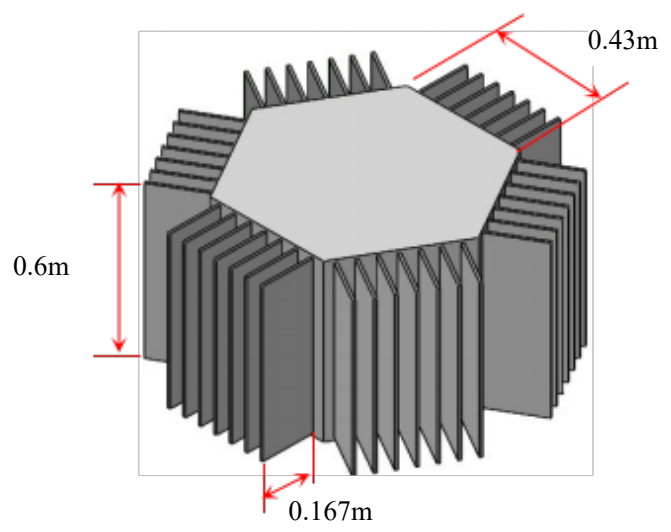

(c)Hexagonal transformer geometry

Figure 2. Conventional rectangular power transformer and proposed transformer geometries

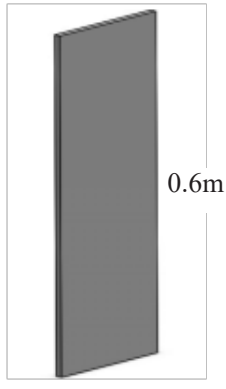

$0.192 \mathrm{~m}$

(a) Rectangular

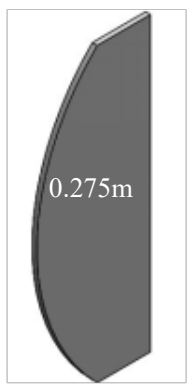

$0.11 \mathrm{~m}$

(b) Semi-circular

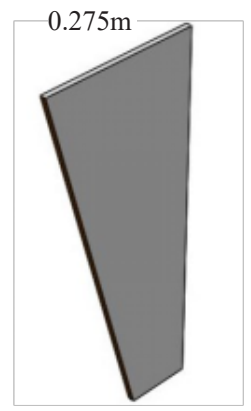

$0.1 \mathrm{~m}$

(c) Trapezoidal

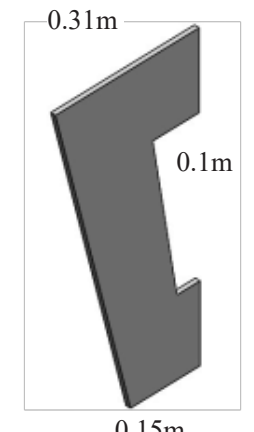

(d) Trapezoidal-perforated

Figure 3. Rectangular fins and the proposed fin shapes

\subsection{Computational model}

\subsubsection{Boundary conditions}

The boundary conditions must be first determined to facilitate the numerical solution of a mathematical model for simulating the selected power transformer. The working conditions, such as the ambient temperature, air velocity, and coil temperature, were determined on the basis of the information provided by the laboratories that is related to those of the ONAN power transformers. Figure 4 shows the 3D computational domain that indicates the boundary conditions 
for all regions. The following are the conditions for the electrical transformer parts, such as oil, body of the transformer, and its fins: (1) Ambient: the external environment of the electrical transformer is represented by static air under 1 atm pressure and different temperatures determined for the study. (2) Core: the inner coils were the surfaces emitting heat, and their minimum and maximum temperatures were determined under the operating conditions of the electrical transformer. (3) Oil: the distance between the windings and the walls of the transformer is filled with oil, and the area is defined with the oil specifications changing with temperature. (4) Side, upper, and lower walls: the top and bottom surfaces and sides of the transformer are defined as the heat transfer surfaces generated with the heat transfer coefficient of the load $\left(0.5 \mathrm{~W} / \mathrm{m}^{2} \mathrm{~K}\right)$. (5) Fins: the sides of the transformer represented by the fins are defined as the diffused surfaces of heat generated to the ambient air with the heat transfer coefficient $\left(1 \mathrm{~W} / \mathrm{m}^{2} \mathrm{~K}\right)$ indicating the transfer of heat by the load. (6) Fluid velocity: Nonslip conditions are applied on all solid surfaces (exterior and interior). The above defined areas have an initial temperature of $298 \mathrm{~K}$.

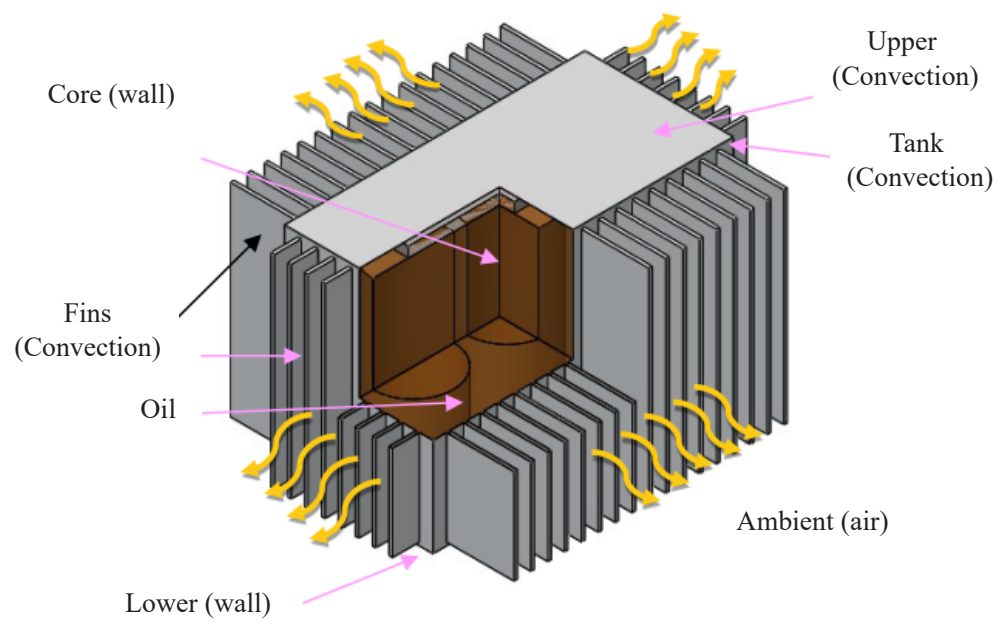

Figure 4. Computational domain for the power transformer

\subsubsection{Governing equation}

Simplification and assumption have been conducted to simplify the model. The solid insulation layers are not created in the geometric model to minimize the complexity and guarantee the grid quality. The movement of the ONAN transformer oil depends on the buoyancy phenomenon. The effect of radiation is negligible. The 3D CFD model calculations for the steady incompressible oil and air flows (oil inside and outside the transformer tank) are based on the three main equations, namely, continuity, momentum, and energy.

Continuity equation

$$
\nabla \cdot \vec{U}=0
$$

Momentum equation

$$
\nabla \cdot(\rho \vec{U}) \vec{U}=-\nabla P+\nabla^{2} \cdot(\mu \vec{U})+g\left(\rho-\rho_{\infty}\right)
$$

Energy equation

$$
\nabla \cdot\left(\rho C_{P} \vec{U} T\right)=\nabla^{2} \cdot(\lambda T)
$$


where $\vec{U}$ is the velocity vector, $P$ is the local fluid pressure, $T$ is the fluid temperature, $\rho$ is the fluid density, $\mu$ is the fluid dynamic viscosity, $g$ is the acceleration of gravity, $C_{P}$ is the fluid specific heat, and $\lambda$ is the fluid thermal conductivity.

The oil properties, such as density, specific heat, viscosity, and thermal conductivity, will change with temperature. The temperature influence of the mineral oil properties is given in the following equations [19]:

$$
\begin{gathered}
\rho=1098.72-0.712 T_{\text {oil }} \\
C_{p}=807.163+3.58 T_{\text {oil }} \\
\mu=8.467 \times 10^{-2}-4 \times 10^{-4} T_{\text {oil }}+5 \times 10^{-7} T_{\text {oil }} \\
\lambda=15.09 \times 10^{-2}-7.101 \times 10^{-5} T_{\text {oil }}
\end{gathered}
$$

\subsection{Numerical solution}

A finite volume method was applied to solve the differential equations for the simulation of the ONAN transformer model. The computational domain was divided into tetrahedron grids to obtain accurate results. The setup must be completed to enable calculations by ANSYS FLUENT (2019 R3) solver and conduct simulation. The steady-state problem was investigated in this study. The SIMPLE algorithm was selected for the solution methods. Velocity and pressure gradients were calculated to solve the momentum equation. In this study, the initial pressure, initial transformer temperature, initial oil temperature, oil properties, and air temperature were defined. Although the flow was natural or in a laminar flow range, the RNG-k- $\varepsilon$ model has an extra term in its equation that significantly improves the accuracy for quickly stressed flows. This model can be actualized to improve the study of the effect of swirl and enhance the accuracy for swirling flows. RNG-k- $\varepsilon$ theory provides a scientifically determined differential equation for viable consistency. The viable utilization of this component does depend on an appropriate treatment of the near-wall region in any case.

\subsection{Grid sensitivity and verification of the numerical model}

Grids are considered one of the most important steps in CFD calculation. High quality grids could improve the result. Given the large scale of the total height and relatively small scale in the radial dimension. In this case, an optimization is needed to obtain an alternative solution for meshing. An alternative way is to reduce the number of grids in the nonsensitive area such as the solid coil, where a low-density quad grid can be used instead of high-resolution grids. The boundary layer near the solid wall should be considered because the transformer oil has a high viscosity. The space between the solid coil and the external transformer body is actually the heat exchanging surface. Highly refined grids are needed in this area to capture the fluid phenomenon. The outer view of the mesh used for the computational model of the hexagonal transformer is shown in Figure 5. The construction of a grid for the body by choosing the appropriate size of the grid that guarantees the accurate results in the shortest possible time is considered one of the important stages of any numerical simulation. Specifically, a balance between the accurate results and their exposure time is necessary to determine the size of the mesh for the body. The improvement of the quality of the solution may necessitate the creation of other grids until the required result accuracy is reached. Meanwhile, the inappropriate use of the grid leads to deviations in the solution. Several tests were conducted for all the proposed configurations of the transformer in this study under fixed conditions, including the air and coil temperatures of 308 and $360 \mathrm{~K}$, respectively. The grid size was changed to ensure that the number ranged between 400,000 and 1,600,000. After performing the simulation, the results of the grid sensitivity test were represented in Figure 6. This figure shows the relationship between the number of selected elements and the average oil temperature. The figure shows all the proposed transformers. The oil temperature of the transformer stabilized after 1,250,000. 


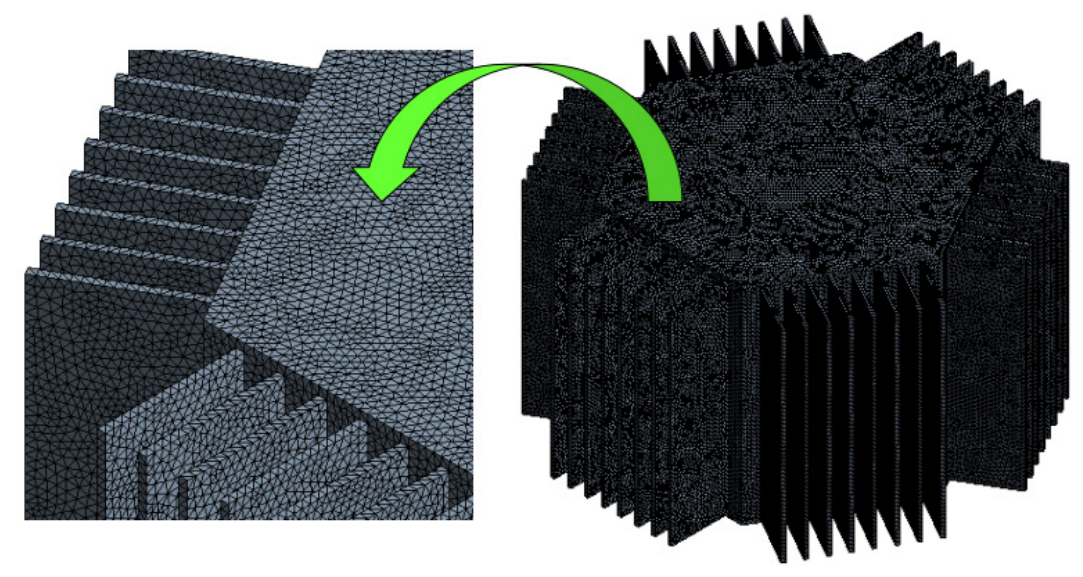

Figure 5. Outer view of the mesh generated. The zoomed image is the hexagonal transformer

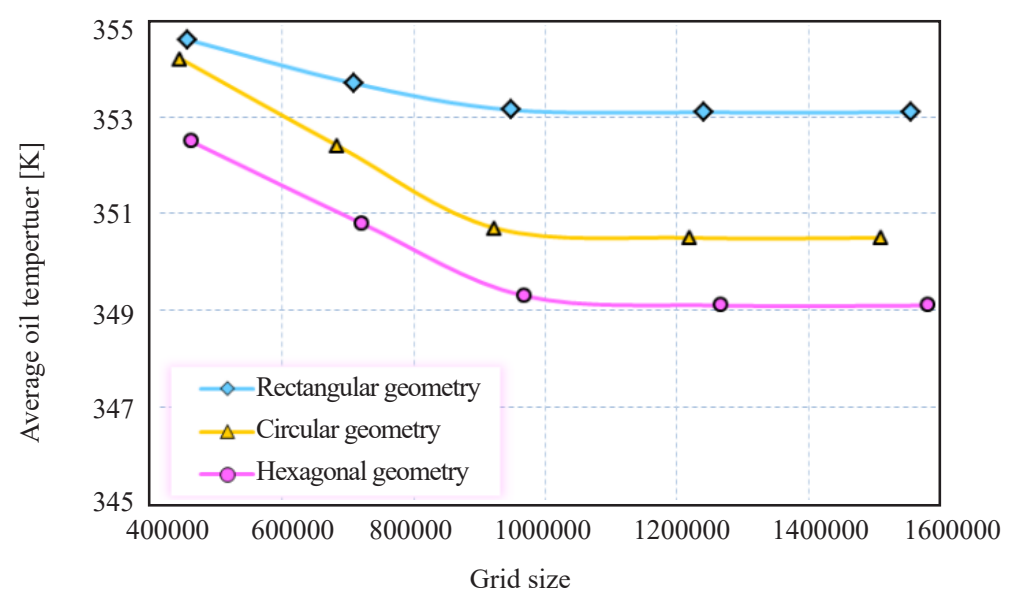

Figure 6. Grid size sensitivity test for the different transformer geometries

The developed numerical model must be validated to reach reliable simulation predicted results. After the numerical model of the traditional ONAN transformer was established and simulated using the ANSYS numerical analysis program within the ambient conditions, the comparison of the simulation results with the experimental data was performed. The numerical simulation results were compared with the experimental data of the practical ONAN transformer, by changing the temperature of the transformer between $335 \mathrm{~K}$ and $375 \mathrm{~K}$ and the ambient air temperature in the transformer between $308 \mathrm{~K}$ and $283 \mathrm{~K}$, under the Iraqi summer and winter weather conditions. The abovementioned effects on the temperature increase of the transformer oil were also discussed. The comparison of the thermal characteristics of the transformer oil with the temperature change of the windings was represented in the summer and winter conditions of Iraq for the practical and numerical studies. Figure 7 shows a comparison between the numerical and the experimental results of the average oil temperature with a change in the temperature of the windings. A clear convergence was observed between the experimental and the numerical results of the average temperature of the transformer oil. We observed a slight maximum local and average difference rate of 7\% and 3\% for the summer season and $6 \%$ and $4 \%$ for winter season. This convergence between the numerically predicted results and the actual outputs of the practical transformation with an error rate within the acceptable range makes this model reliable. The difference is due to two main reasons. First, some losses in the experimental side are observed, and the accuracy of the measuring devices that used is poor. Second, the numerical side is based on a set of assumptions for performing numerical simulations. 


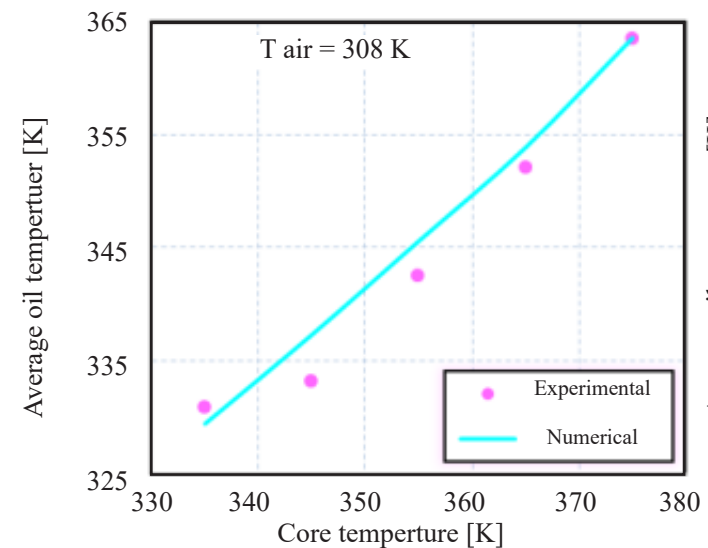

(a) Summer season

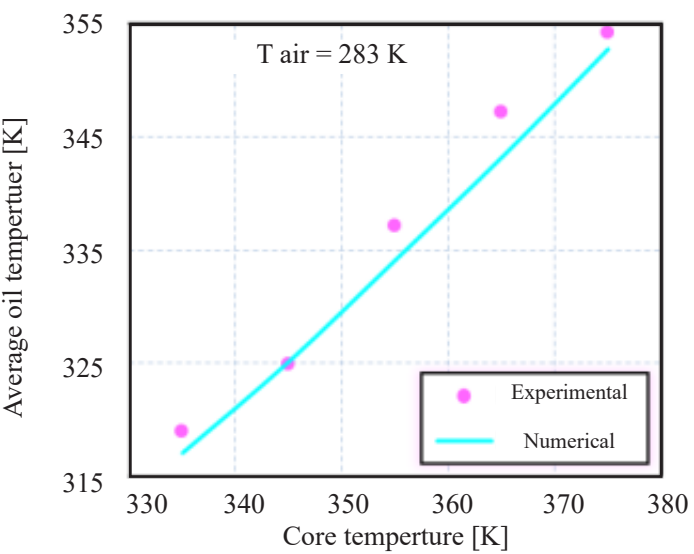

(b) Winter season

Figure 7. Comparison between the experimental and the numerical results of the variation of the average oil and core temperature

\section{Experimental methodology}

\subsection{Experimental test rig}

A $250 \mathrm{kVA}$ distribution transformer (Figure 1), which is widely used in the Iraqi electricity network, is selected as a case study. The transformer dimensions are tabulated in Table 1. A three-phase distribution transformer (250 kVA, $11 /$ $0.416 \mathrm{kV}$, core type, oil immersed, and natural convection) has been assembled and provided with the necessary sensors for testing in the electrical industries in Baghdad / Iraq. The experimental setup of the ONAN transformer similar to the thermal test in the laboratory is shown in Figure 8. The transformer consists of coils, a core assembly, three copper coils, and a steel core linking them. These items are immersed in the transformer oil contained in the transformer body equipped with fins to increase the heat transfer area. The transformer oil plays two important roles: a cooling medium transfers the heat generated in coils and core into outer walls to dissipate it to the outside; and electric insulator. The available data indicated that the heat generated in the transformer in a full load situation is $1000 \mathrm{~W}$ from each coil and $500 \mathrm{~W}$ from the core. This generated heat must be dissipated to maintain the temperature of oil at a certain accepted level.

After the ONAN transformer was assembled, and all first electricity laboratory tests were conducted, the losses of power transformer were successfully measured. The transducer was transferred to a special laboratory to be subjected to loads on the coils and monitor the oil temperature over time. The electrical transformer was prepared for thermal testing according to the following steps:

1. The work (short circuit) of the poles of the electrical transformer coils of the low voltage (LV) and the connection of the high voltage (HV) with wires provide the voltage that is determined by the examiner. The amount of voltage applied is related to the high temperature of the electrical coils, thereby causing an increase in the temperature of the oil.

2. The transformer is provided with a set of eight type-K thermocouples distributed as follows:

- Four thermocouple sensors are installed on both sides of the tank facing the LV and HV at the top and bottom.

- One thermocouple sensor is placed inside the tank to measure the oil temperature.

- Three thermocouple sensors with dispensers with jugs containing an amount of oil are placed $1 \mathrm{~m}$ from the sides of the transformer to measure the ambient temperature.

Digital thermometers were used to measure the temperature from the calibrated thermocouples. The thermometer has recording capabilities and computer interface with an accuracy of $0.1 \%+0.5^{\circ} \mathrm{C}$. After completing the abovementioned two steps, tests are carried out according to IEC60076 by setting the specified amounts of voltages. We manually recorded the temperature sensor readings for each operation for $1 \mathrm{~h}$ until the oil temperature stabilizes. The operating hours must not be less than seven consecutive hours. The readings are fixed to a list prepared by the laboratory to convert it into a program to map the rate of oil temperature rise over time. 


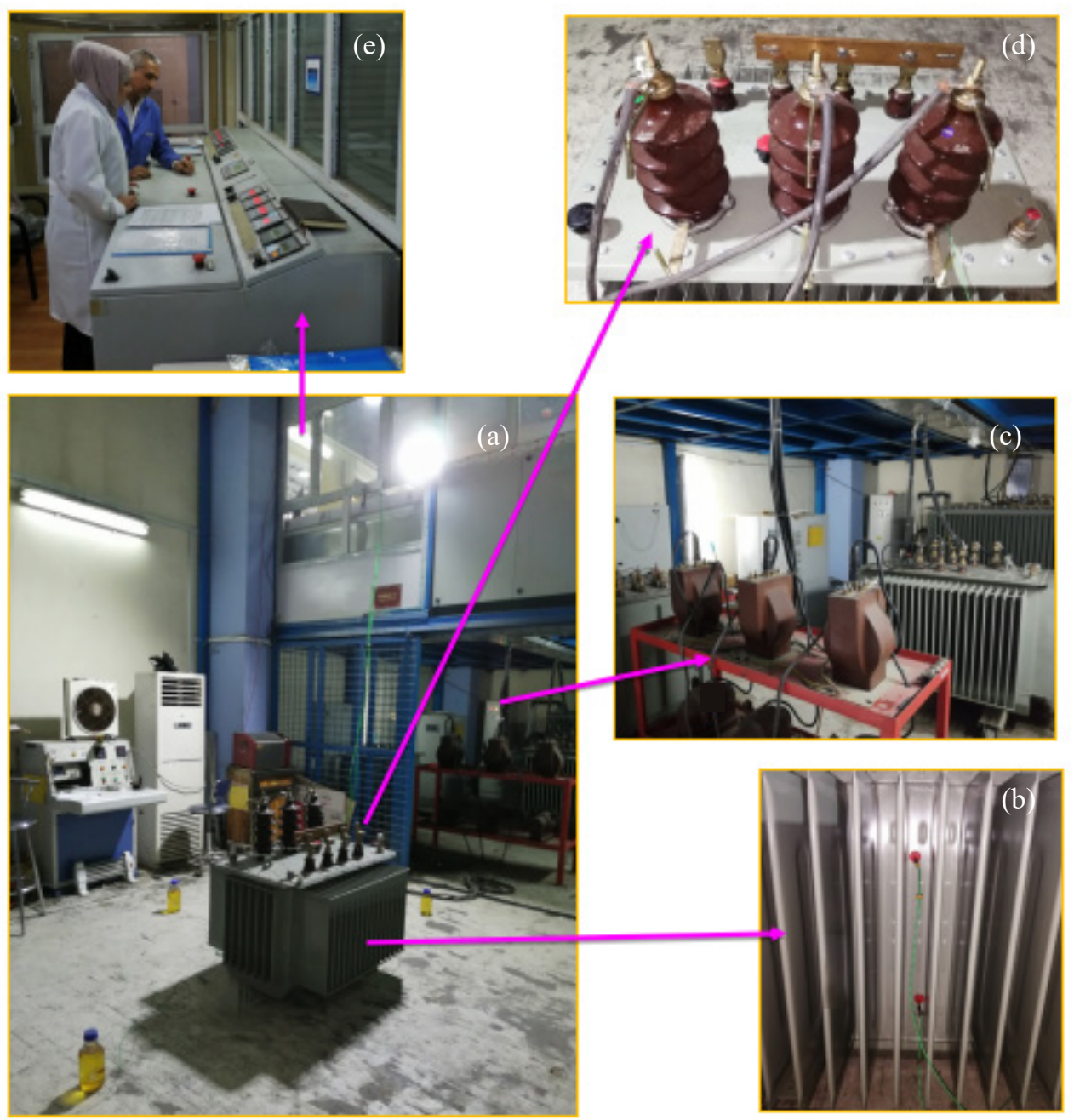

Figure 8. Experimental setup of the ONAN transformer similar to the thermal test in the laboratory: (a) Electrical power transformer; (b) Section of fins with temperature sensors installed;

(c) Processing unit; (d) Connection to the voltage supply; (e) Control and monitoring unit

\subsection{Experimental procedure}

After the electrical transformer were prepared, the tests related to the current study were conducted. These tests include temperature tests for the atmosphere $10^{\circ} \mathrm{C}$ and $35^{\circ} \mathrm{C}$, i.e. making the electrical transformer in winter and summer time for Iraqi weather conditions. After the air temperature in the laboratory was determined (for example, winter), a specific value was dropped from the voltages to the transformer. We recorded the temperature sensors for $1 \mathrm{~h}$ until the temperature of the oil has stabilized. The number of test hours must not be less than seven consecutive hours. In the following day, the experiment was repeated at the same voltage to check the recorded readings. The rechecking was conducted by assigning another value to the voltage at the same selected air temperature. The test was performed in the same manner. The process of projecting five voltages with the same ambient temperature was repeated to obtain an average oil stable temperature and the transformer temperature. The above-mentioned method of examination was repeated, but with a temperature of the ambient (summer) and with the same voltage values used in the first examination. 


\section{Results and discussion}

The effects of the proposed changes on the geometry of the transformer and the shape and arrangement of its fins are analyzed and discussed. The first part of the section shows the effect of the transformer geometry on the cooling performance. The suitable transformer geometry that achieves the optimal thermal performance is then determined. The second part shows the effect of fin shapes on the performance of the transformer to the optimal transformer geometry chosen in the first part. The third part tests the effect of fin arrangement on the circumference of the transformer. This part determines the effect of the optimal shape of the fin that was identified from the second part on the performance of the transformer.

\subsection{Effect of transformer geometry}

As previously explained, the maximum hot spot temperature of the transformer must be reduced to increase its efficiency and longevity. The comparison of the proposed designs with the conventional design of the transformer will be based on that temperature. Figure 9 shows the temperature oil contours for the different geometries, namely, rectangular, circular, and hexagonal, under an air temperature of $308 \mathrm{~K}$ and core (coil) temperature of $365 \mathrm{~K}$. The temperature of oil at the upper section is higher compared with that at the lower section for all geometries. Moreover, the temperature gradually changes between two sections. A decrease in the temperature of the oil at the middle of the top surface of the transformer can be observed due to two main reasons. First, the top surface is in a direct contact with the surrounding air, and the oil layer in this area is thin. The heat transfer at that region is high, and a decrease in the oil temperature is observed. The second reason is due to the location of the files inside the transformer and the method of dispersing the heat. The heat that dissipates toward the sides of the transformer is greater than that disperses to the top surface of the coil, thereby causing an increase in the oil temperature at the sides. Accordingly, the density of the oil varies, and the less dense oil rises to the top, taking the shape surrounding the coil. The temperature of the oil on the sides of the upper surface must be as high as possible. The hexagonal transformer has a better performance compared with the three transformer geometries. This result is due to the lower oil temperature at the hexagonal transformer shape than those of the circular and rectangular transformer shapes. The temperature of the circular transformer shape is less than that of the rectangular one. Hence, the proposed designs (hexagonal and circular) are better than the original one (rectangular shape). For instance, the temperature differences of oil are $22^{\circ} \mathrm{C}, 24^{\circ} \mathrm{C}$, and $25^{\circ} \mathrm{C}$ for rectangular, circular, and hexagonal geometries, respectively. This result indicates that changing the shape of the transformer plays an important role in improving the heat transfer despite maintaining the surface area of the transformer. The proposed designs contributed to the increase in heat dispersion to the atmosphere, especially the hexagonal transformer shape, due to the increase in the transformer sides while reducing the number of fins in one side. This situation leads to the nonaccumulation of heat in the middle fins compared with that in the original transformer. The location of the coil in the hexagonal transformer faces several sides, thereby allowing for a great heat exchange. 


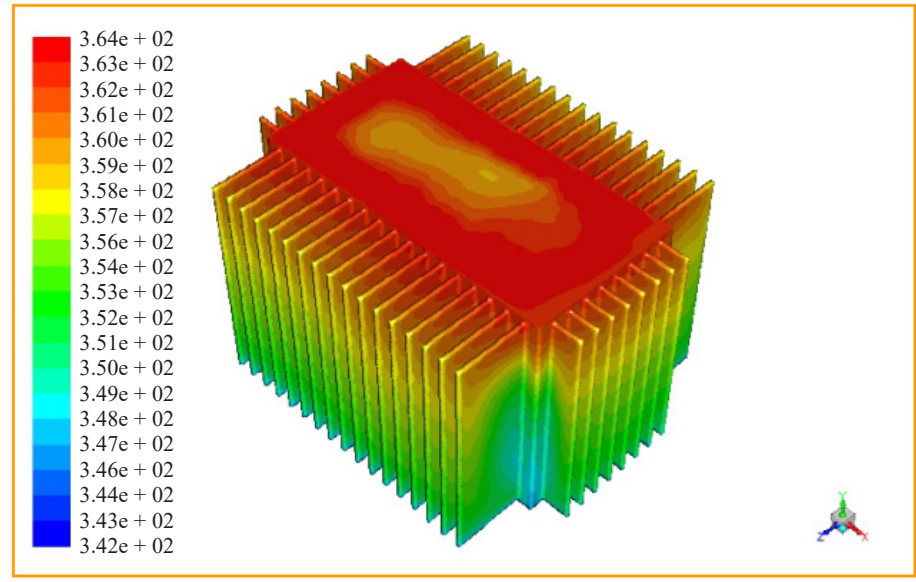

(a) Rectangular geometry

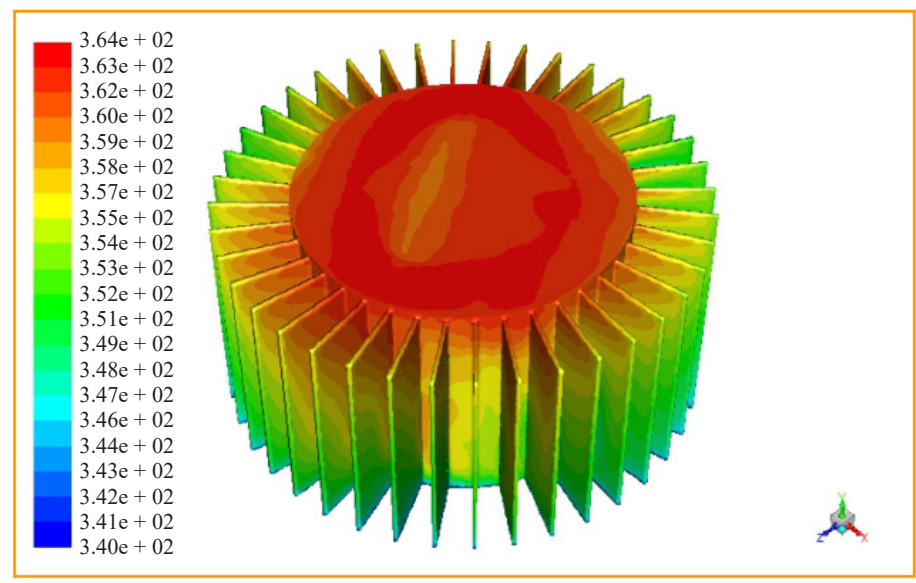

(b) Circular geometry

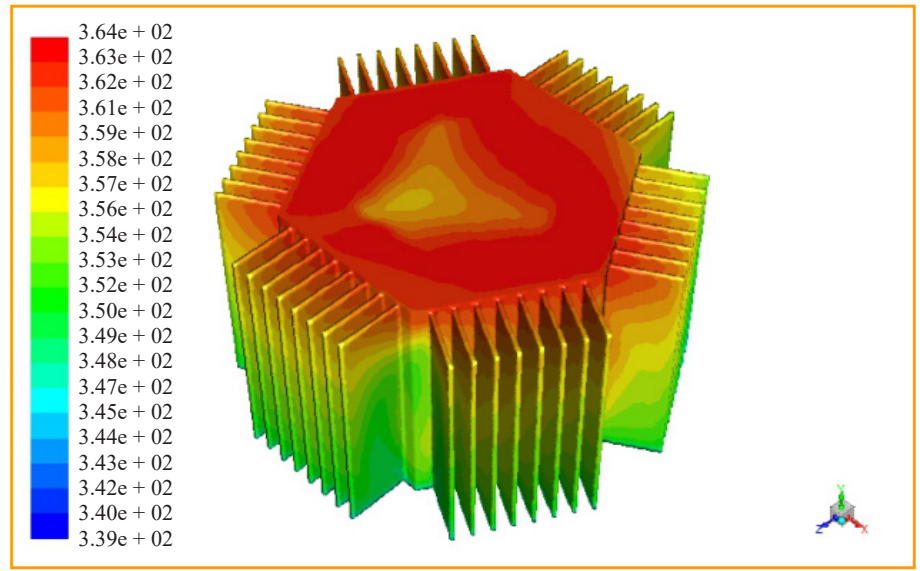

(c) Hexagonal geometry

Figure 9. Temperature oil contour for the different transformer geometries

The oil temperature values of the transformers increase to high and critical values when the ambient temperature increases. This situation is one of the serious problems that cause failure of the electrical transformers, especially when operating in a hot climate and extreme load conditions. The aforementioned condition may result in the collapse of the transformer and stop its operation. Figure 10 indicates the variation of the average oil temperature of the rectangular, circular, and hexagonal geometries with outside air temperature at the coil temperature of $363 \mathrm{~K}$. The air temperature 
of $328 \mathrm{~K}$ was chosen as the maximum temperature to simulate the maximum air temperature in summer for Iraqi ambient air. The figure shows that the average oil temperature for the different transformer geometries increases with the increase in the outside air temperature due to the decrease of the heat dissipation to the outside as a result of the decreasing temperature difference. This situation leads to the accumulation of heat in the oil. The transformer with a circular shape demonstrated a decreased oil temperature of 3.4\% compared with that with a rectangular shape. Meanwhile, the percentage of reduction in the temperature of the oil reached $4.7 \%$ when the hexagon transformer was used. Figure 11 shows the variation of the average oil temperature of the rectangular, circular, and hexagonal geometries with inside coil temperature at air temperature of $308 \mathrm{~K}$. This figure shows that the average oil temperature of the different transformer geometries increases with the increase in the inside coil temperature due to the decrease in the heat dissipation to the outside as a result of the decreasing temperature difference. This situation leads to the accumulation of heat in the oil. The temperature changes of the oil for all the proposed shapes of transformers have a converging value at constant air temperature. This phenomenon is due to the rate of heat transfer from the coils to the oil that depends on the difference in the density of the oil. The transfer of heat from the oil to the ambient air is through the fins and with a high heat transfer rate. The surface area for heat transfer from coils to oil is less than that for heat transfer from the oil to the air. Accordingly, the oil temperature changes for all shapes with converging values. However, the oil temperature of the hexagonal transformer is the lowest compared with those of the other shapes. Figures 10 and 11 demonstrate that air temperature is an influencing factor on oil temperature. The oil is considered a coil insulator and a heat transfer to be dissipated from the coils to the surrounding environment. The effects of the high temperature of the oil are as follows: the density of the oil changes, and a buoyancy phenomenon occurs. Figure 12 shows the variation of the average oil density with outside air temperature for the three types of transformer geometries. The density behavior of the oil is completely opposite to the behavior of the oil temperature. This condition corresponds to the logic. The figure exhibits that the average oil density when using a hexagonal shape is larger than that when rectangular and circular shapes are utilized and decreased with increasing outside air temperature for all different transformer geometries. Heat flux is one of the important determinants in evaluating the performance of electrical transformers. Figure 13 shows the variation of the heat flux at the transformer outer walls with outside air temperature for the three types of transformer geometries. This heat flux provides an indication about the amount of heat that can be dissipated to the surrounding air. The amount of heat transferred when using hexagonal geometry is larger than those when rectangular and circular geometries are utilized. This phenomenon is due to the high temperature difference between the outer surface of the fins and the ambient air temperature. In all the cases under study, a linear decrease in the heat flux is observed with the increase in air temperatures.

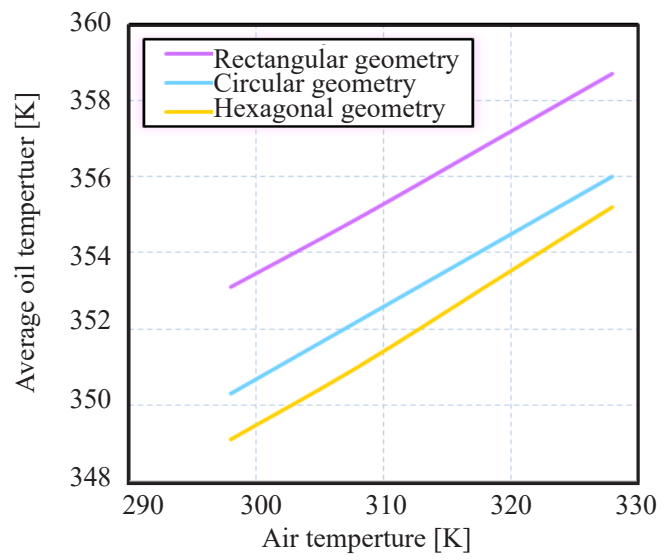

Figure 10. Variation of the average oil temperature with outside air temperature for the different transformer geometries 


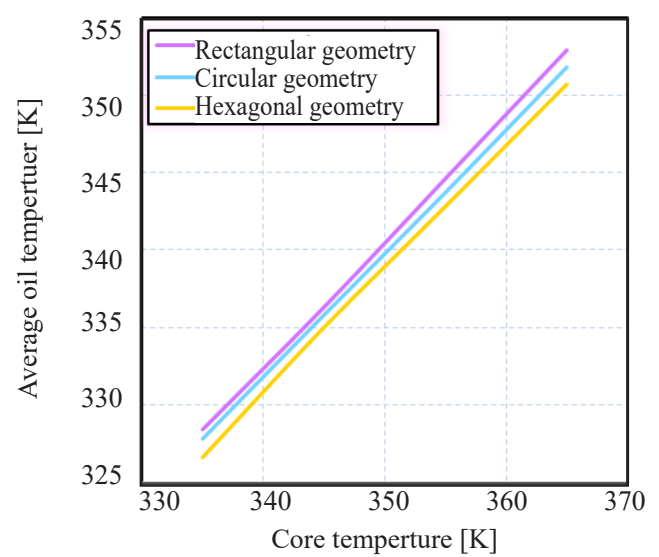

Figure 11. Variation of the average oil temperature with inside core (coil) temperature for the different transformer geometries

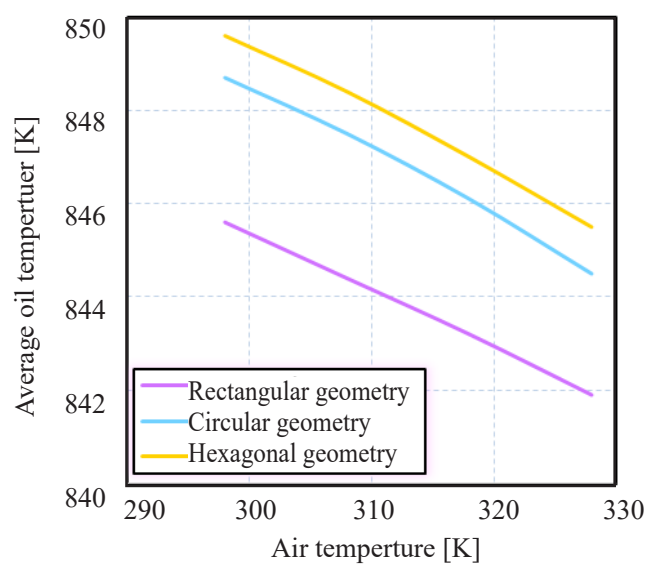

Figure 12. Variation of the average oil density with outside air temperature for the different transformer geometries

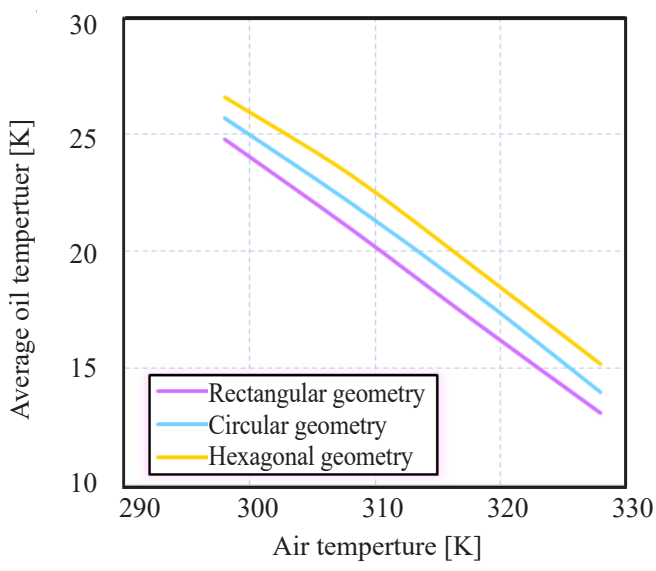

Figure 13. Variation of the average removed heat flux with outside air temperature for the different transformer geometries

\subsection{Effect of fin shapes}

The results from the previous section showed that the hexagonal shape of the transformer has a better performance compared with the other geometries. The performance of the hexagon transformer was evaluated relative to the effect of 
the distance between the fins. A slight improvement in the performance was observed when the distance was increased from $4 \mathrm{~cm}$ to $5 \mathrm{~cm}$. The distance of $5 \mathrm{~cm}$ will be the distance between the fins for this case. This section tests the effect of the fin shape on the thermal performance of the hexagonal transformer. Three different fin shapes, namely, traditional rectangular, semicircular, and trapezoidal, will be tested. Figure 14 shows the temperature oil contour of the hexagonal transformer geometry with rectangular, semicircular, and trapezoidal fins shapes. A decrease in oil temperature was observed in the trapezoidal fin compared with the traditional one (rectangular). This phenomenon was due to the increased heat dissipation in the upper region of the transformer resulting from an increase in the surface area in that region compared with the bottom region. Such phenomenon was also due to the maximum heat transfer resulting from a high difference in temperature between the oil and the ambient air. The upper region of the transformer showed the highest oil temperature compared with the other transformer regions due to the difference in oil density. Accordingly, a larger surface area can be acquired at that region to obtain high heat dissipation with ambient air. This result was obtained when the trapezoidal fin was used.

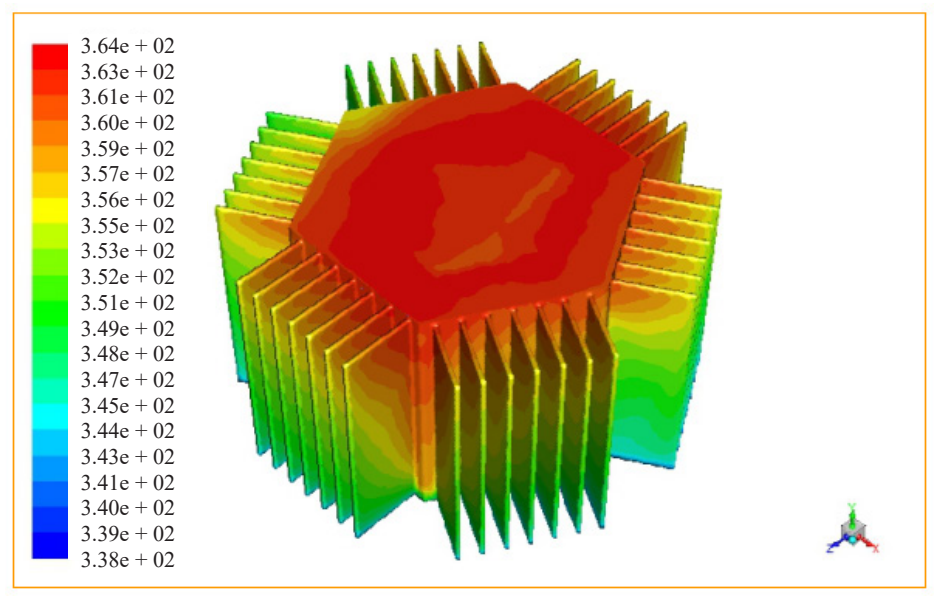

(a) Hexagonal geometry (Rectangle fins)

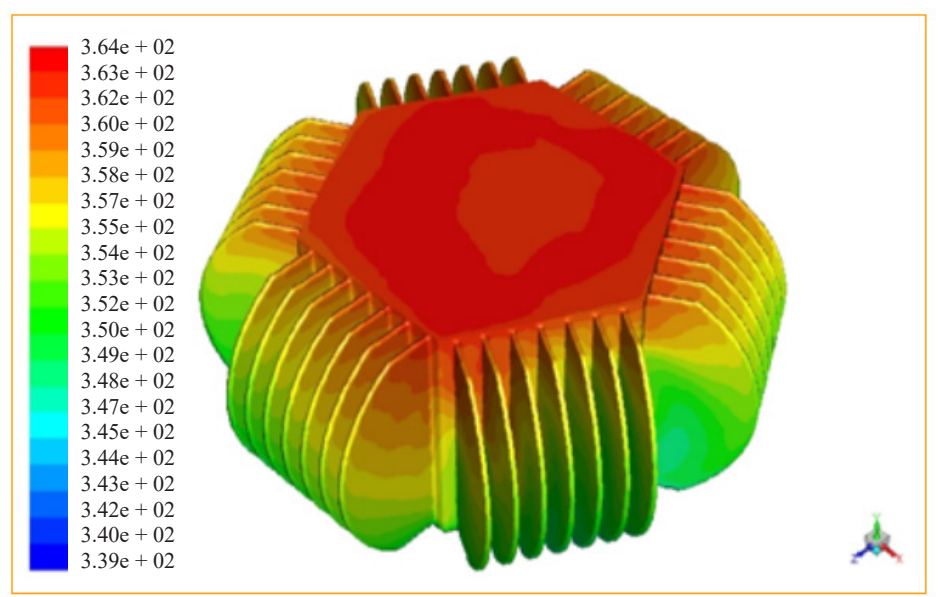

(b) Hexagonal geometry (Semi-circle fins) 


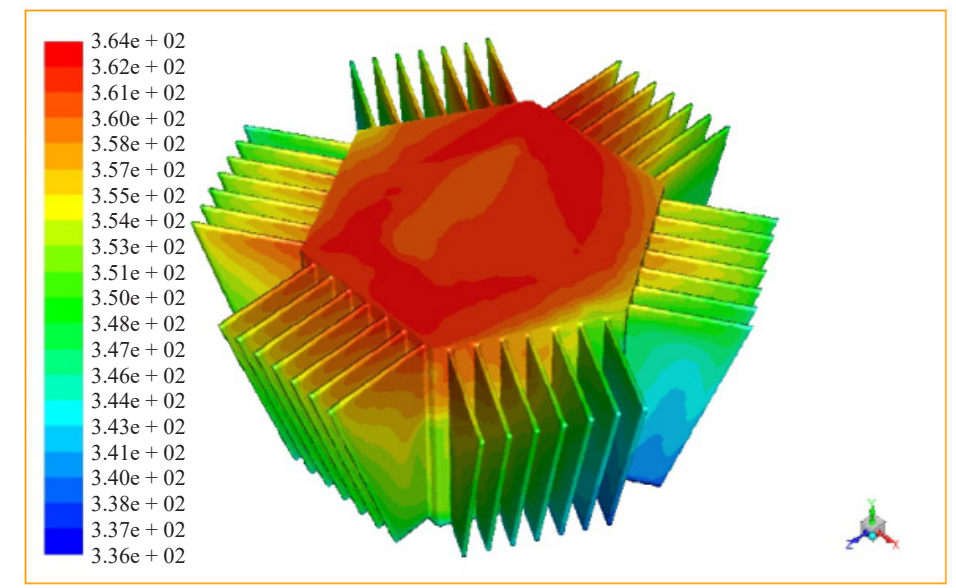

(c) Hexagonal geometry (Trapezoidal fins)

Figure 14. Temperature oil contour of the hexagonal transformer geometry with different fins shapes

Figure 15 shows the variation of the average oil temperature with outside air temperature of the hexagonal transformer geometry with different fins shapes (rectangle, semicircle, and trapezoidal). A direct proportion of the oil temperature with the ambient temperature of all the proposed fins for the transformer is observed. The lowest oil temperature is observed for the trapezoidal fin, followed by the rectangular and semicircular fins, as indicated in the previous figures.

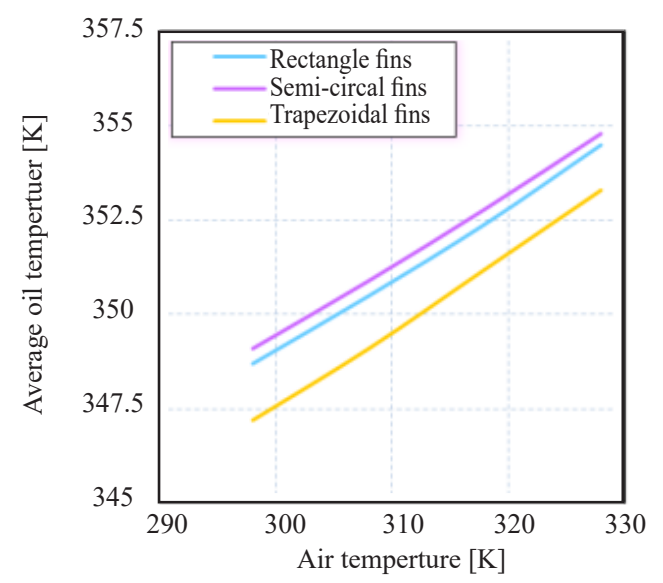

Figure 15. Variation of the average oil temperature with outside air temperature of the hexagonal transformer geometry with different fins shapes

\subsection{Effect of fin arrangements}

The previous section indicated that the change in the shape of the fin has a significant role in increasing the heat transfer rates. The trapezoidal shape of the fin provides a good heat transfer between the transformer oil and the ambient air. Accordingly, the hexagonal transformer with trapezoidal fins was chosen. This section will focus on the study of the effect of the arrangement of fins on the thermal performance of the selected transformer. Figure 16 shows the temperature oil contour of the hexagonal transformer geometry for the trapezoidal fins with several arrangements. This study presents two models for the trapezoidal fin. The first model is the alteration of the asymmetric fin heights by repeatedly changing the heights of the fins. The second model includes perforation. The result showed that the asymmetric fin heights of the trapezoidal fin demonstrated a better performance compared with that of the original trapezoidal fins because the minimum oil temperature decreased by $3^{\circ} \mathrm{C}$. An optimal thermal performance was also 
obtained when the trapezoidal fin was perforated. This phenomenon was attributed to the cutting area of the trapezoidal fin that was offset by an area added to the fin itself to ensure that the surface area specified for the fin was preserved. Moreover, the same amount of oil was preserved inside the cavity of the fin. The area harvested from the fin created additional ventilation for the fin, and it ensured the circulation of the oil inside the annular cavity, thereby increasing the cooling rate of the oil. The low temperature of the trapezoidal fin arrangement decreased by $9^{\circ} \mathrm{C}$.

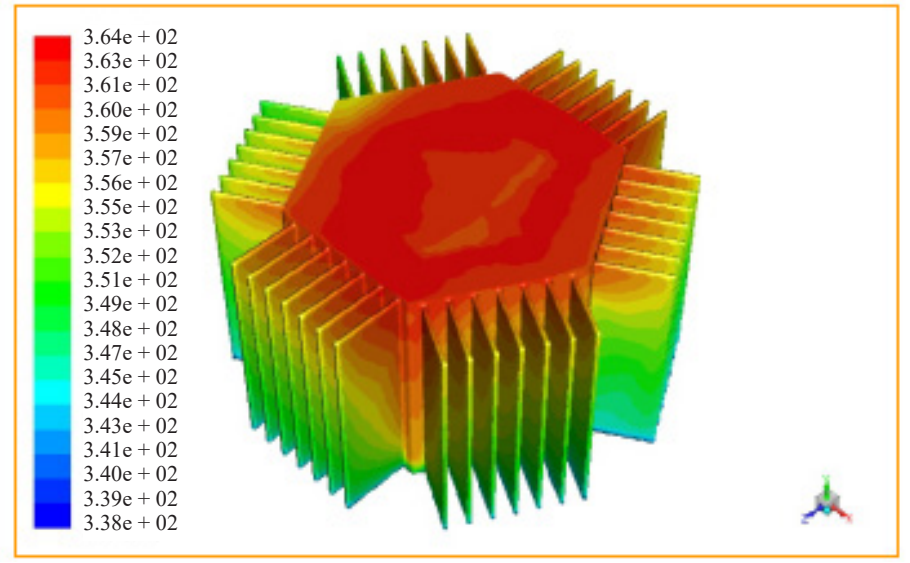

(a) Hexagonal geometry (Trapezoidal fins)

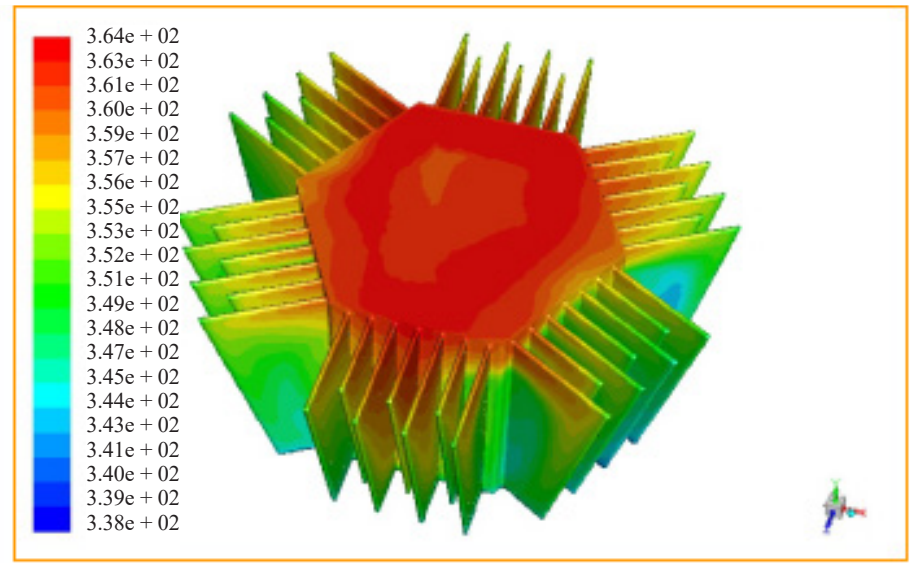

(b) Hexagonal geometry (Trapezoidal fins) (Asymmetric fin heights)

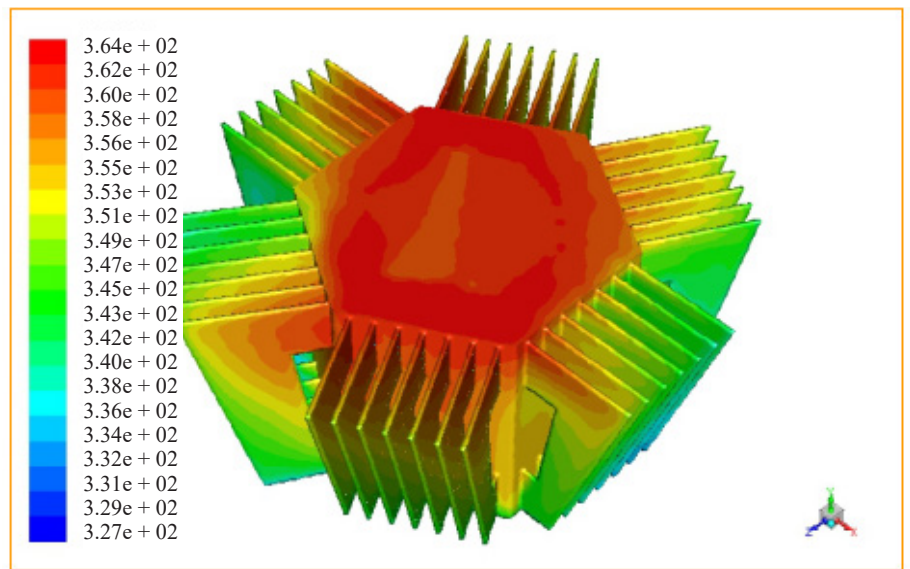

(c) Hexagonal geometry (Trapezoidal fins) (Perforated)

Figure 16. Temperature oil contour of the hexagonal transformer geometry for the trapezoidal fins with several arrangements 
Figure 17 demonstrates the variation of the average oil temperature with outside air temperature of the hexagonal transformer geometry for the trapezoidal fins with several arrangements. The previous explanations indicated that a constant behavior of the oil temperature with the ambient temperature was observed for all models presented in the study. The above figure exhibits that the oil temperature follows the same behavior as it increases with the increase in ambient temperature. A low oil temperature was also observed when the perforated trapezoidal fins were used for the hexagonal transformer and for the reasons mentioned above.

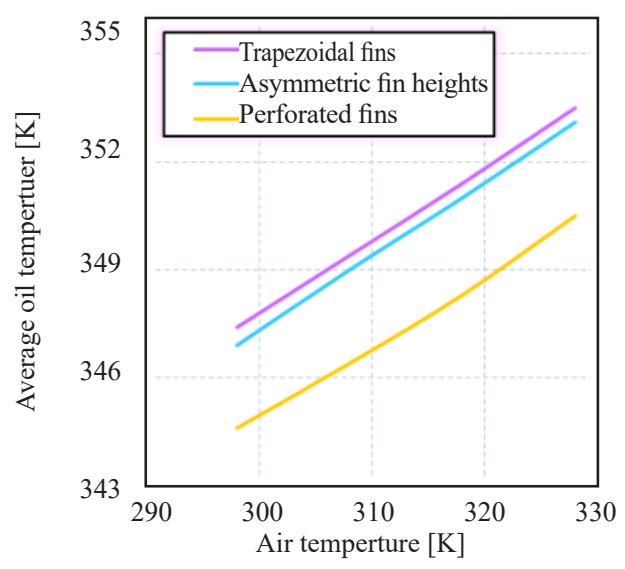

Figure 17. Variation of the average oil temperature with outside air temperature of the hexagonal transformer geometry for the trapezoidal fins with several arrangements

\subsection{Overall optimization}

In this section, the overall performance improvement of the hexagonal transformer with a perforated trapezoidal fin comparison to the traditional rectangular transformer will be discussed. Figure 18 shows the variation of the average oil temperature with outside air temperature to compare between the traditional rectangular and the new design transformers. It can be noted that the relationship between oil temperature and air temperature for both configurations is linear and directly proportional. Furthermore, this figure demonstrates an effective decrease of the oil temperature for the hexagonal transformer with a perforated trapezoidal fin reached $12 \%$ compared with the conventional rectangular transformer. the reason behind that is the increase the heat transfer surface area with surrounding due to the use of perforated trapezoidal fin and the hexagonal transformer shape. Hence, it can be concluded that the hexagonal transformer with a perforated trapezoidal fin gives the best performance among the series of improvements on the shape of the transformer and the shapes and arrangements of the fins. 


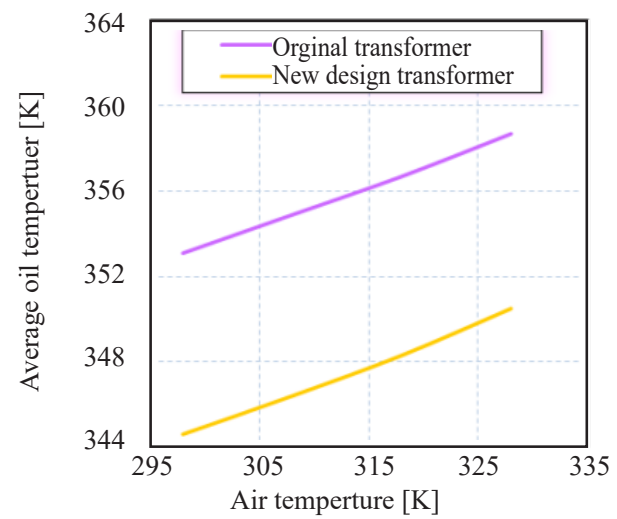

Figure 18. Variation of the average oil temperature with outside air temperature between the original and the new design transformers

\section{Conclusions}

Power transformers are considered one of the essential equipment for electrical power distribution and transmission systems. In this work, new changes to the geometry of the transformer and the shape and arrangement of fins were proposed to improve the cooling performance of the ONAN power transformer by reducing the maximum oil temperature. Numerical prediction analysis was performed by using 3D finite volume technique and CFD. The numerical simulation model was validated with the experimental work. A clear convergence was observed between the experimental and the numerical results of the average temperature of the transformer oil. The simulation model was considered reliable when the maximum error was approximately $4 \%$. The following main important conclusions can be drawn:

1. The geometry of the transformers plays an important role in improving the heat transfer despite their similar surface areas. The proposed circular and hexagonal shapes reduced the average oil temperature by $3.4 \%$ and $4.7 \%$, respectively, compared with the traditional (rectangular) transformer shape.

2. Fin shape played a major role in the heat transfer process, and its effect was dependent on the large surface area of the hot portions. The trapezoidal fin showed the lowest average oil temperature, followed by the rectangular and the semicircular fins.

3. The asymmetric fin heights of the trapezoidal and perforated trapezoidal fins contributed to the improvement in the cooling performance of the transformer. A good thermal performance was also obtained when the trapezoidal fin was perforated.

4. An effective decrease in the oil temperature of the oil was observed when the hexagonal transformer with a perforated trapezoidal fin reached $12 \%$ compared with the conventional rectangular transformer.

\section{Acknowledgments}

The authors of this paper wish to express their deep gratitude and sincere thanks to Mustansiriyah University (www. uomustansiriyah.edu.iq) in Baghdad / Iraq.

\section{Conflict of interest}

The authors declare no conflict of interest. 


\section{Nomenclatures}

$\mathrm{Cp}=$ specific heat, $\mathrm{J} / \mathrm{kgK}$

$\mathrm{g}=$ gravitational accelerating, $\mathrm{ms}^{-2}$

$\mathrm{H}=$ height of the channel, $\mathrm{mm}$

$\mathrm{h}=$ heat transfer coefficient, $\mathrm{W} / \mathrm{m}^{2} \mathrm{~K}$

$\mathrm{I}=$ current, ampere

$\mathrm{P}=$ pressure, $\mathrm{Pa}$

$\mathrm{T}=$ temperature, $\mathrm{K}$

$T_{\text {oil }}=$ oil temperature, $\mathrm{K}$

$\mathrm{U}=$ velocity, $\mathrm{m} \mathrm{s}^{-1}$

$\mathrm{V}=$ voltage, volt

\section{Greek Symbols}

$\rho=$ density, $\mathrm{kg} \mathrm{m}^{-3}$

$\rho_{\infty}=$ air density, $\mathrm{kg} \mathrm{m}^{-3}$

$\mu=$ dynamic viscosity, $\mathrm{N} \mathrm{s} / \mathrm{m}^{2}$

$\lambda=$ thermal conductivity, $\mathrm{W} / \mathrm{mK}$

\section{References}

[1] Morteza Mikhak-Beyranvand, Jawad Faiz and B. Rezaeealam, "Thermal analysis of power transformer using an improved dynamic thermal equivalent circuit model," Electric Power Components and Systems, vol. 47, no. 18, pp. 1598-1609, 2019.

[2] Bizhan Mehrvarz, Fatemeh Bahadori and Saeed Zolfaghari Moghaddam, "Heat transfer enhancement in distribution transformers using $\mathrm{T}_{\mathrm{i}} \mathrm{O}_{2}$ nanoparticles," Advanced Powder Technology, vol. 39, no. 2, pp. 221-226, 2019.

[3] Alex Skillen, Alistair Revell, Hector Iacovides, and WeiWu, "Numerical prediction of local hot-spot phenomena in transformer windings," Applied Thermal Engineering, vol. 36, pp. 96-15, 2012.

[4] G. W. Swift, S. E. Zocholl, M. Bajpai, J. F. Burger, C. H. Castro, S. R. Chano, F. Cobelo, P. de Sá, E. C. Fennell, J. G. Gilbert, S. E. Grier, R. W. Haas, W. G. Hartmann, R. A. Hedding, P. Kerrigan, S. Mazumdar, D. H. Miller, P. G. Mysore, M. Nagpal, R. V. Rebbapragada, M. V. Thaden, J. T. Uchiyama, S. M. Usman, J. D. Wardlow and M. Yalla, “Adaptive transformer thermal overload protection," IEEE Transactions on Power Delivery, vol. 4, no. 6, pp. 516-521, 2001.

[5] Inmaculada Fernández, Fernando Delgado, Félix Ortiz, Alfredo Ortiz, Cristina Fernández, Carlos J. Renedo and Agustín Santisteban, "Thermal degradation assessment of Kraft paper in power transformers insulated with natural esters," Applied Thermal Engineering, vol. 104, pp. 129-138, 2016.

[6] Kaike Sa Teles Rocha Alves, Michel Hell, Fernando Luiz Cyrino Oliveira and Eduardo Pestana de Aguiar, “An enhanced set-membership evolving participatory learning with kernel recursive least squares applied to thermal modeling of power transformers," Electric Power Systems Research, vol. 184, pp. 106334, 2020.

[7] M.A. Taghikhani and A. Gholami, "Prediction of hottest spot temperature in power transformer windings with nondirected and directed oil-forced cooling," Electrical Power and Energy Systems, vol. 31, no. 7-8, pp. 356-364, 2009.

[8] S Anishek, Sony R, Jayadeep Kumar J and Pradeep M Kamath, "Performance Analysis and optimisation of an oil natural air natural power transformer radiator," Procedia Technology, vol. 24, pp. 428-435, 2016.

[9] Marina A. Tsili, Eleftherios I. Amoiralis, Antonios G. Kladas and Athanassios T. Souflaris, "Power transformer thermal analysis by using an advanced coupled 3D heat transfer and fluid flow FEM model," International Journal of Thermal Sciences, vol. 53, pp. 188-201, 2012.

[10] Mustafa S. Mahdi, Anees A. Khadom, Hameed B. Mahood, Mahmood Abdul Razak Yaqup, Jammal M. Hussain, Khalid I. Salih and Hussein A. Kazem, "Effect of fin geometry on natural convection heat transfer in electrical distribution transformer: Numerical study and experimental validation," Thermal Science and Engineering Progress, vol. 14, pp. 100414, 2019.

[11] F. Bachinger and P. Hamberger, "Thermal measurement of an ester-filled power transformer at ultralow 
temperatures: steady state," Procedia Engineering, vol. 202, pp. 130-137, 2017.

[12] Ebrahim Hajidavalloo and Mohamad Mohamadianfard, "Effect of sun radiation on the thermal behavior of distribution transformer," Applied Thermal Engineering, vol. 30, no. 10, pp. 1133-1139, 2010.

[13] Bharadwaj R. Sathyanarayana, Gerald T. Heydt and Michael L. Dyer, "Distribution transformer life assessment with ambient temperature rise projections," Electric Power Components and Systems, vol. 37, no. 9, pp. 1005-1013, 2009.

[14] Jon Gastelurrutia, Juan Carlos Ramos, Gorka S. Larraona, Alejandro Rivas, Josu Izagirre and Luis del Río, "Numerical modelling of natural convection of oil inside distribution transformers," Applied Thermal Engineering, vol. 31, no. 4, pp. 493-505, 2011.

[15] M. Abdolzadeh, A. Khoshabi and M. A. Mehrabian, "Thermal analysis of an electric power transformer inside an enclosure-a case study," International Journal of Ambient Energy, vol. 38, no. 3, pp. 250-258, 2017.

[16] Muhammad Farhan, Muhammad Saad Hameed, Hafiz Muhammad Suleman and Muhammad Anwar, "Heat transfer enhancement in transformers by optimizing fin designs and using nanofluids," Arabian Journal for Science and Engineering, vol. 44, pp. 5733-5742, 2019.

[17] Olivier Arguence and Florent Cadoux, "Sizing power transformers in power systems planning using thermal rating," Electrical Power and Energy Systems, vol. 118, pp. 105781, 2020.

[18] N. El Wakil, N.-C. Chereches and J. Padet, "Numerical study of heat transfer and fluid flow in a power transformer," International Journal of Thermal Sciences, vol. 45, no. 6, pp. 615-626, 2006.

[19] Ruohan Gong, Jiangjun Ruan, Jingzhou Chen, Yu Quan, Jian Wang and Cihan Duan, "Analysis and experiment of hot-spot temperature rise of $110 \mathrm{kV}$ three-phase three-limb transformer", Energies, vol. 10, no. 8, pp. 1-12, 2017.

[20] Nelu-Cristian Chereches, Monica Chereches, Livia Miron and Sebastian Hudisteanu, "Numerical study of cooling solutions inside a power transformer," Energy Procedia, vol. 112, pp. 314-321, 2017.

[21] Min-gu Kim, Sang Moon Cho and Joong-Kyoung Kim, "Prediction and evaluation of the cooling performance of radiators used in oil-filled power transformer applications with non-direct and direct-oil-forced flow," Experimental Thermal and Fluid Science, vol. 44, pp. 392-397, 2013.

[22] A. Gamil, A. Al-Abadi, M. Schiessl, F. Schatzl and E. Schlücker, "Improvements on thermal performance of power transformers: Modelling and testing," in 2019 6th International Advanced Research Workshop on Transformers (ARWtr), 2019, pp. 13-18.

[23] R. M. Hannun, S. H. Hammadi and M. H. Khalaf, "Heat transfer enhancement from power transformer immersed in oil by earth air heat exchanger," Thermal Science, vol. 23, no. 6, pp. 3591-3602, 2019.

[24] M. I. Hasan, "Improving the cooling performance of electrical distribution transformer using transformer oil-based MEPCM suspension," Engineering Science and Technology, an International Journal, vol. 20, no. 2, pp. 502-510, 2017. 